\title{
GENERATION OF A 30 GHZ TRAIN OF BUNCHES USING A MAGNETIC SWITCH-YARD
}

\author{
B. Autin, R. Corsini, CERN, CH 1211 Geneva 23, Switzerland
}

A possible technique of generation of high-frequency drive beam for a linear collider consists in creating electron bunches in a battery of guns and merging them in a magnetic circuit (switch-yard) at the end of which all the bunches are aligned on a common line with the right spacing. Two types of scheme are discussed. The properties of the structure in terms of orbit dispersion, focusing and bunch lengthening due to finite momentum spread and emittance are analyzed.

\section{INTRODUCTION}

The CLIC project of a $\mathrm{e}^{+} \mathrm{e}^{-}$linear collider is based on the two-beam accelerator scheme [1]. The electron and positron beams are accelerated in $30 \mathrm{GHz}$ accelerating structures, the needed $\mathrm{rf}$ power being generated by the interaction of a high power relativistic electron beam (the Drive Beam), running parallel to the main linac, with appropriate $\mathrm{rf}$ structures resonant at $30 \mathrm{GHz}$ (transfer structures). Each drive beam (one for the positrons, and one for the electrons) has an energy of $3 \mathrm{GeV}$ and is composed of $2.5 \mu \mathrm{C}, 12 \mathrm{~ns}$ long electron bursts, at the same $\sim 2 \mathrm{kHz}$ repetition rate of the main beam. Each burst is composed of typically four trains of 22 short $(\sigma \sim 1 \mathrm{~mm})$ bunchlets. The bunch charge is $30 \mathrm{nC}$, and the bunches are spaced by 1 $\mathrm{cm}$. This spacing corresponds to the $30 \mathrm{GHz}$ frequency of the transfer structures. While it seems feasible to generate and accelerate single $30 \mathrm{nC}$ bunchlets in an S-band photocathode rf gun + booster, with the desired bunch length (possibly by using magnetic pulse compression), the correct spacing between bunchlets cannot be directly achieved. Furthermore, the beam loading limits the number of bunchlets that can be accelerated in such a system during the $12 \mathrm{~ns}$ duration of each burst. Therefore, one needs more of these sources to generate the full drive beam, and a method of combining the pulses together at the right spacing. The proposed technique consists of using one array of 11 electron guns for each drive beam [2]. Each gun delivers 2 bunchlets per train, and each operates at a different energy from 25 to $50 \mathrm{MeV}$. The bunchlets are then merged in a single trajectory using a dipole magnet and undergo further acceleration downstream. This bunch recombination scheme could be tested in future (possibly with only two lines) in the CLIC Test Facility (CTF) [3].

The general shape of a switch-yard is shown in figure 1. The optical structure of the channels is presented for two types of scheme christened $\mathrm{C}$ and $\mathrm{S}$. The $\mathrm{C}$-scheme is presently contemplated for the big machine and the Sscheme seems to be more appropriate for the test facility. It has also to be noted that such structures can be of interest all along the drive beam each time the beam has to be bent while satisfying the condition of isochronicity.

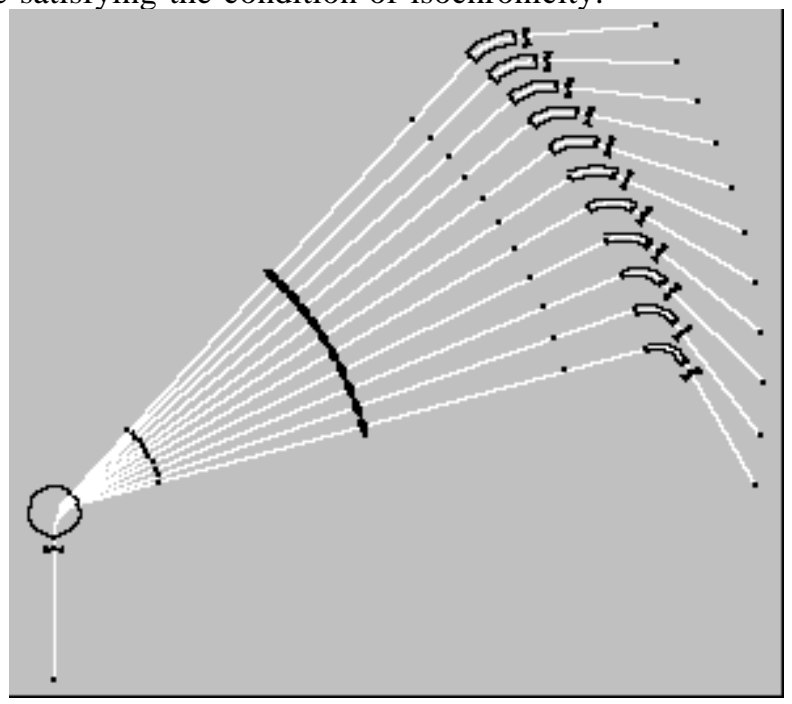

Figure 1. Orbits in a switch-yard

$$
\text { II C- and S-schemes }
$$

Two symmetric configurations of switch-yard channels are compared in this paper. The $\mathrm{C}$-scheme is symmetrical with respect to an axis and the deflections of the two bending magnets add up. The $\mathrm{S}$-scheme is symmetrical with respect to a point and the two deflections are of opposite sign so that the input and output trajectories are parallel. Their schematic structure is shown in figure 2 . The bending magnets are represented by white circles. The shape is indeed circular for the magnet in which all the bunches are merged before exiting on a common straight section. 


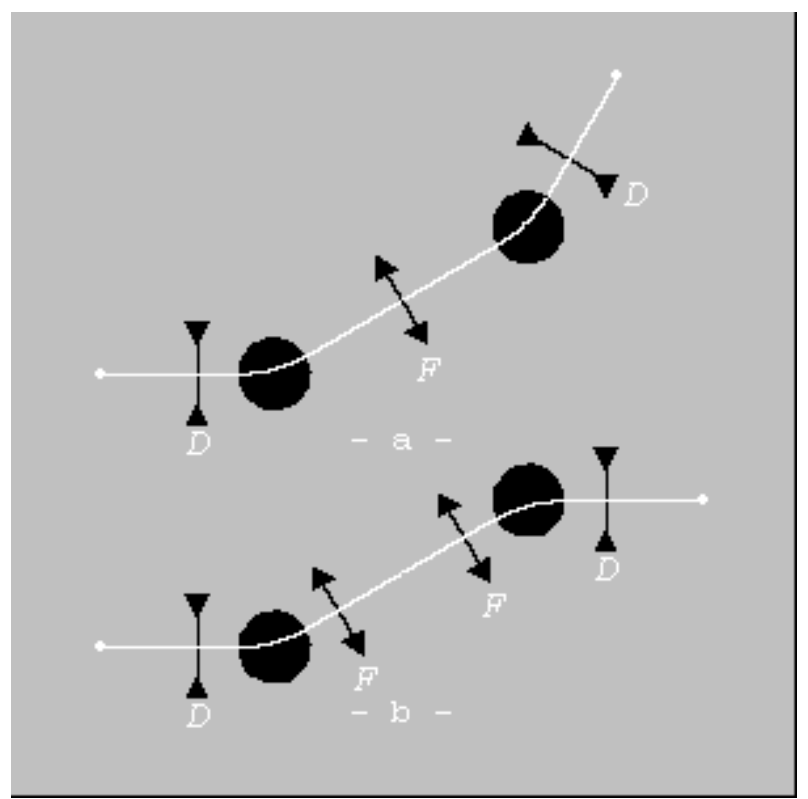

Figure 2. C-channel (a) and S-channel (b).

When few beams have to be combined, a static dipole may advantageously be replaced by a radio frequency dipole. In this configuration, the bunches receive the same deflections but with an alternating sign. The other dipole is actually a sector magnet proper to each channel. The focusing structure is idealized by thin lenses which permit algebraic calculations and an analytical formulation of the properties of the schemes. Detailed analysis using thick lenses is beyond the scope of this study. For reasons which will be discussed in the next chapter, the focusing is performed with singlets in the C-case and doublets in the Scase.

\section{Optics of a switch-yard channel}

A magnetic switch-yard is easily analyzed when the particles are traced backwards from the straight section where the bunches are merged to the photo-injectors. The bunch motion is traditionally split into the motion of its centre of gravity and the oscillations of the other particles about the centre of gravity.

\section{A. Orbits}

The orbits (see figure 1) are the trajectories of the centers of gravity of the bunches. They start with a common straight section and are then discriminated by a spectrometer whose magnetic field is constant and shape is a circle of radius $R$. The trajectories in the spectrometer are arcs of circles orthogonal to the contour circle; their radius is proportional to the particle momentum. With respect to a reference orbit of momentum $p$ and deflection $\phi$, a neighbour orbit of momentum $p+\Delta p$ is radially displaced at a distance $l$ from the end of the spectrometer by the quantity $D \Delta p / p$ where the orbit dispersion $D$ is given by:

$$
D=(R+l) \sin \phi
$$

This separation is needed for placing focusing elements. In order to keep the whole system compact, the separation is to be small and the space reserved to the quadrupoles is comparable to the spectrometer radius and of the order of $20 \mathrm{~cm}$ which is compatible with permanent magnet technology. The second separation to be achieved concerns the photo-injectors at the end of the channel. In a symmetric configuration, it is determined by the distance $2 d$ between the two spectrometers. For the C- and Sscheme they are equal to

$$
\begin{aligned}
& s_{C}=(R+d) \sin \phi+R(1+\cos \phi) \\
& s_{S}=2(R+d) \sin \phi
\end{aligned}
$$

The $\mathrm{C}$-scheme is more practical than the $\mathrm{S}$-scheme because of its fan shape but, for a separation of $30 \mathrm{~cm}$ at the location of the injectors, the distance between the spectrometers is of the order of $5 \mathrm{~m}$ in both cases.

\section{B. Focusing}

The switch-yard channel is dispersive in its very principle but it connects two straight sections where both the orbit dispersion $D$ and its longitudinal derivative $D^{\text {, }}$ must be zero. The dispersion increase in the spectrometers is always the same but keeps rising in the $\mathrm{C}$-scheme up to the F-lens where it is bent back towards the second spectrometer whereas, in the S-scheme, it is immediately focused to the center of symmetry. Such orbit dispersion patterns impose the focal length of the F-lens:

$$
\begin{aligned}
& f_{C}=\frac{R+d}{2} \\
& f_{S}=\frac{(R+l)(d-l)}{R+d}
\end{aligned}
$$

In the $\mathrm{S}$-case, $l$ is the distance from the end of the spectrometer to the F-lens. It turns out that the focusing is much stronger for the $\mathrm{S}$ - than for the $\mathrm{C}$-scheme. The variation of the orbit dispersion is shown in figures 3 and 4 .

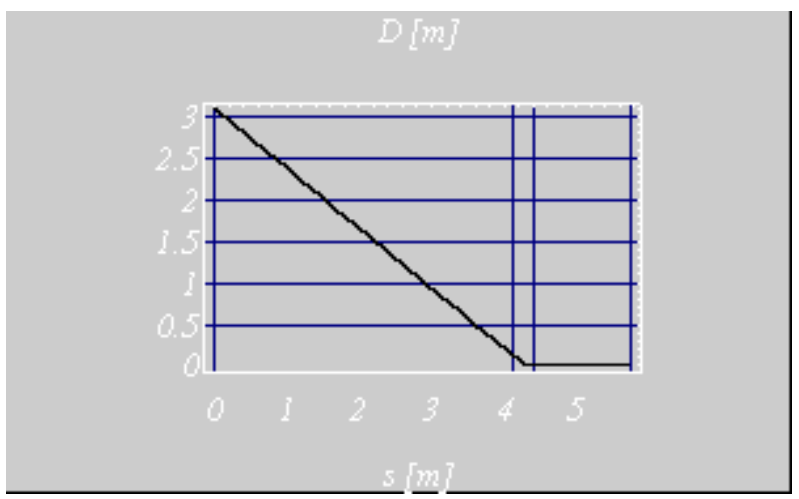

Figure 3. Orbit dispersion in a half C-channel. 


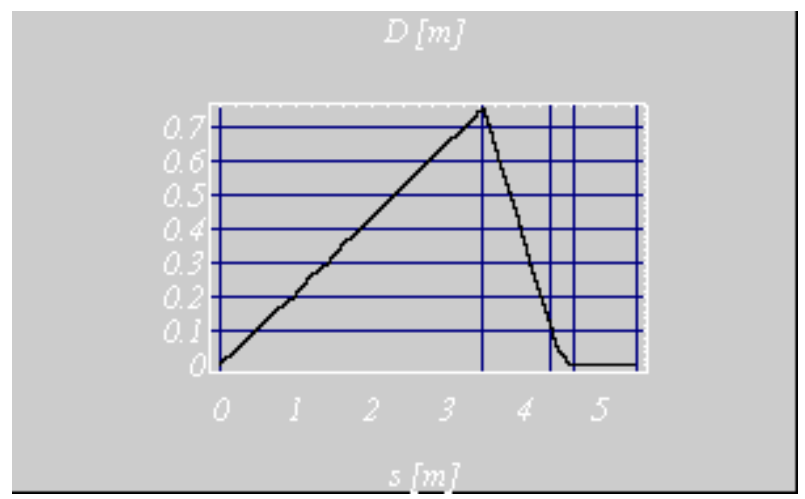

Figure 4. Orbit dispersion in a half S-channel.

The horizontal focusing being fixed, the vertical focusing is provided by D-lenses located in zero dispersion regions, i.e. the input and ouput straight sections. The structure is made of alternating $\mathrm{D}$ - and F-lenses in the Cscheme and of two symmetric doublets in the S-scheme. The conditions

$$
\begin{aligned}
& \beta_{h}-\beta_{v}=0 \\
& \alpha_{h}+\alpha_{v}=0
\end{aligned}
$$

at the end of the channel are canonical to match a FODO cell directly or via a quarter wave-length transformer [4]. The two unknowns of the problem are the focal length of the D-lens and the distance from the D-lens to the matching point. With the help of a symbolic program, factorizations can be performed and the physical solutions turn out to be uniquely determined though they are solutions of a fourth degree equation. The $\beta$-functions are somewhat arbitrary since the periodic lattice which surrounds the channel is not a priori defined but rather fitted to the switch-yard. The $\beta$-functions at the symmetry point which serve as input values to the $\beta$-tracking have been chosen so as to minimize the $\alpha$-function at the end point to facilitate the betatron matching. The variations of the $\beta$-functions are shown in figures 5 and 6.

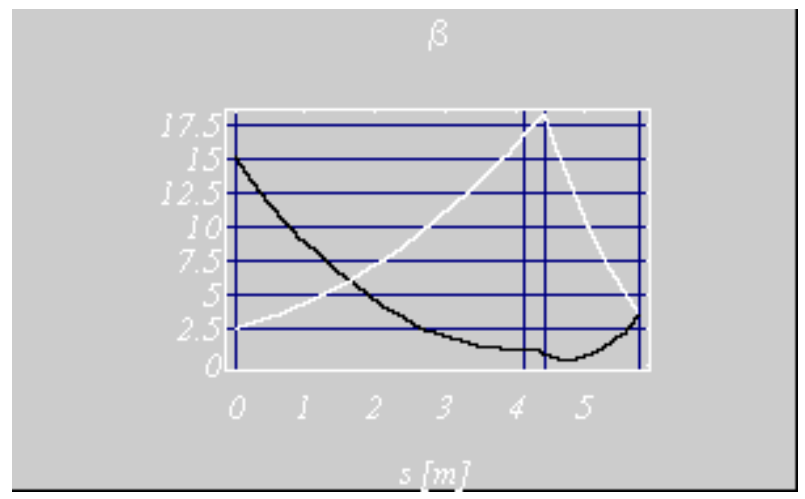

Figure 5: $\beta$-functions in a half $\overline{C \text {-channel. }}$

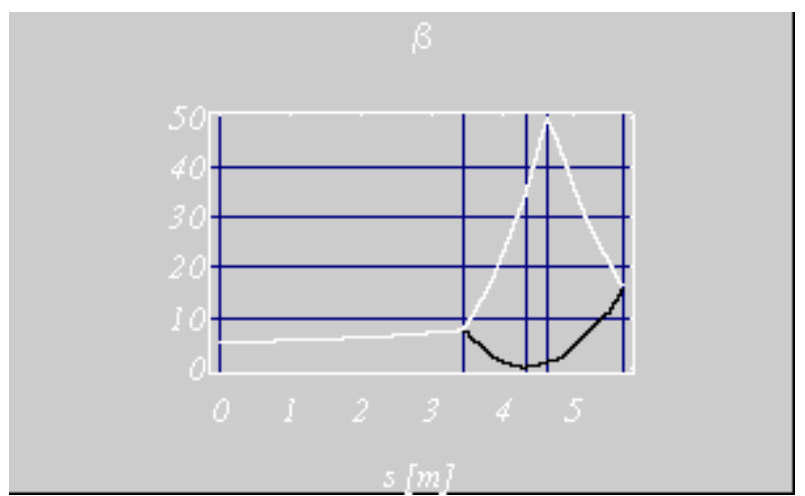

$\overline{\text { Figure 6. } \beta \text {-functions in a half }} \overline{\text { S-channel. }}$

In each bunch the particles have a finite momentum spread and it is indispensable to superimpose on the quadrupolar component of the lenses a sextupolar component

$$
f^{\prime}=D f
$$

where $f$ ' is the radial derivative of the quadrupole focal length. This correction can only be performed in the $\mathrm{F}$ lenses where the orbit dispersion is non-zero and the chromatic aberrations, yet not fully compensated, remain tolerable.

\section{Path length}

In the generation of the train of bunches the distance between bunches is determined by the trigger of the injectors and by the path length of the various channels. A bunch lengthening due to the transverse emittance of the beam and to its momentum spread must also be kept under control.

When two particles of same momentum and longitudinal position enter a spectrometer, they leave it at different longitudinal positions distant by

$$
d z=(x-R \dot{x}) \sin \phi
$$

if their radial distance is $x$ and their horizontal angles differ by $x^{\prime}$ at the input. From this stand point, an S-scheme is self compensated at least to first order. Position and angle are related by the Courant and Snyder invariant and it can be shown that for a given emittance ellipse of area $\varepsilon$, the bunch lengthening varies from zero to a maximum value which depends on $\phi, R, \varepsilon$ and the $\beta$-function at the end of the dipole.

The change in path length is related to the relative momentum error through the path length dispersion $D_{l}$

$$
\Delta z=D_{l} \frac{\Delta p}{p}
$$

whose expression in a dipole is

$$
D_{l}=\rho(\phi-\sin \phi)
$$

In contrast to the case of the emittance dependence, the path length always increases in a dipole because the radius of curvature $\rho$ and the deflection $\phi$ have the same sign. $D_{l}$ can nevertheless be controlled by shifting the quadrupoles radially. A displacement $\Delta x$ produces a kick 


$$
\Delta \phi=\frac{\Delta x}{f}
$$

and a change in path length $D \Delta \phi$. The focal distance $f$ of the F-lens has a new expression because the extra kick modifies the orbit dispersion

$$
\begin{aligned}
& f_{C}=\frac{(R+d) \sin \phi}{2 \sin \phi+\Delta \phi} \\
& f_{S}=\frac{(R+I)(d-l) \sin \phi}{(R+d) \sin \phi+(d-I) \Delta \phi}
\end{aligned}
$$

\section{CONCLUSION}

The characteristics of optical structures which can be inserted in zero dispersion regions with a controlled isochronicity have been described in the framework of a linear model which gives the basic parameters in an analytical way. The simulation of higher order and space charge effects is not reported here but has been done and confirms the feasibility of a magnetic switch-yard.

\section{REFERENCES}

[1] H. Braun et al., "CLIC - A Compact and Efficient High Energy Linear Collider", This Conference.

[2] W. Schnell, "The Drive Linac for a Two-Stage Linear Collider", CERN-LEP-RF/88-89 and CLIC Note 85.

[3] R. Bossart et al., "CLIC Test Facility Developments and Results", This Conference.

[4] K.L. Brown and R.V. Servranckx, AIP Conference Proceedings, 127, pp 62-138 (1985) 